\title{
Swimming Monofin Optimization
}

\author{
Marco A. Luersen* \\ INSA de Rouen, Saint Etienne du Rouvray, 76801, France, and CEFET-PR, Curitiba, Brazil \\ Rodolphe Le Riche ${ }^{\dagger}$ \\ Ecole des Mines de Saint Etienne, Saint Etienne, 42023, France
}

Didier Lemosse

INSA de Rouen, Saint Etienne du Rouvray, 76801, France

\author{
Olivier Le Maître ${ }^{\S}$ \\ Eric Breier ${ }^{\natural}$ \\ Université d'Evry Val d'Essonne, Evry, 91020, France \\ Breier S.A.S., Saint Avé, 56890, France
}

\begin{abstract}
Monofins provide swimmers with an efficient alternative to the standard pair of fins. For example, all short and long distance human swimming records have been established using monofins. Current monofin design is mostly empirical, so the objectives of this work are to analyze monofin propulsion through coupled fluid-structure simulation and to optimize its flexural stiffness distribution. The optimization process maximizes the propulsive power provided by the monofin with a constraint on the total expended power. In order to be able to carry out the optimization of the coupled fluid-structure system which is numerically costly to evaluate, the following simplifications are proposed: (i) a twodimensional unsteady, inviscid and incompressible fluid flow is considered; (ii) the swimmer is composed of linear articulated segments, whose kinematics is imposed and identified from experimental data; (iii) the monofin is represented by rigid bars linked by torsional springs. For various allowable swimmer powers optimal $2 \mathrm{D}$ stiffness distributions are obtained using the Globalized and Bounded Nelder-Mead algorithm. Finally, an identification procedure is described to translate the optimal $2 \mathrm{D}$ stiffness distributions into $3 \mathrm{D}$ thickness profiles for a given monofin planform shape.
\end{abstract}

\section{Introduction}

Monofins already provide the most efficient way of swimming for human beings. However, it is expected that further progress can be achieved because today's monofin design is empirical and studies in aquatic locomotion modes and oscillating hydrofoils show that more efficient swimming systems exist. Fish like tuna, mackerel, sharks and marine mammals have propulsive efficiency greater than $90 \%$ at high swimming speed in calm waters. ${ }^{1}$ Similar efficiencies have been observed for artificial oscillating hydrofoils. ${ }^{2,3}$ Previous works have been devoted to describing the physics and physiology of fin-swimming. ${ }^{4,5}$ The objective of the current work is to propose a rationale for designing monofins.

\footnotetext{
*Doctorate Student, LMR - Laboratoire de Mécanique, Avenue de l'Université.

${ }^{\dagger}$ CNRS Permanent Research Associate, Mechanical and Materials Department, 158 Cours Fauriel.

¥Assistant Professor, LMR - Laboratoire de Mécanique, Avenue de l’Université.

$\S$ Assistant Professor, Laboratoire de Mécanique et d'Energétique d'Evry, 40 Rue du Pelvoux.

『Breier Fin Company Manager, 13 Rue Marcel Dassault.
} 


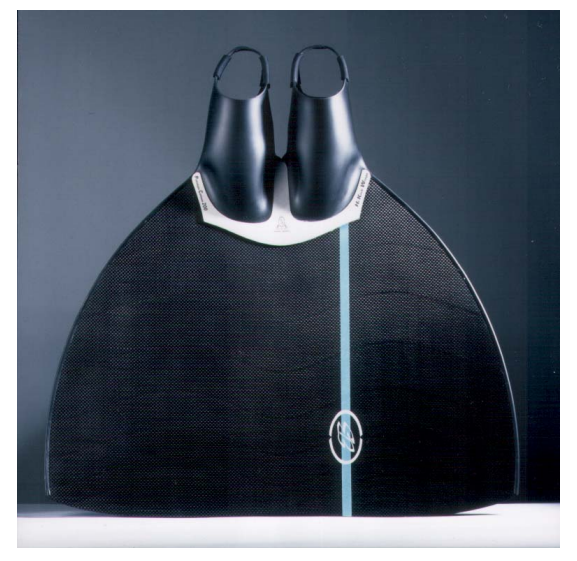

Figure 1. Carbon monofin (Breier(c)).

The simulation of the swimmer and monofin system is very complex: the flow is unsteady, it interacts with the fin which is a composite structure in dynamic motion with large displacements. There are many works related to the fluid-structure interaction and its impact on structural design (e.g., Kvamsdal et al. ${ }^{6}$ ), but the numerical costs of typical fluid-structure models do not allow their optimization which requires a large number of evaluations.

In order to carry out the monofin optimization, some model simplifications are made in the first part of the article: (i) a two-dimensional unsteady, inviscid and incompressible fluid flow without separation is assumed; (ii) the swimmer is represented by linear articulated segments whose kinematics is identified from experimental swimmer data and imposed; (iii) the monofin is represented by rigid bars linked by torsional springs with large rotations allowed. Due to the small monofin thickness and flow features, the sheet vortex fluid model presented in Le Maître et al. ${ }^{7}$ is used.

In the second part of the article, the propulsive power provided by the monofin is maximized with a constraint on the maximum total power expended by the swimmer. The design variables are the spring rigidities. The optimization problem is solved by the Globalized and Bounded Nelder-Mead (GBNM) algorithm. ${ }^{8}$

The flexural stiffness distribution obtained from the two-dimensional optimization is finally translated into a three-dimensional structure. The mapping can be seen as an identification procedure where the "experience" is a 2D bars system whose behavior is approximated by a $3 \mathrm{D}$ finite element model of the fin. The equivalence between the two models can be sought in terms of static behavior, dynamic behavior, or a mix of static and dynamic behaviors. The advantages of static equivalence is that the load cases can be taken from the 2D flow simulation and large displacements analyses are available. However, it neglects the fin inertia. On the contrary, the modal dynamic identification accounts for both flexural and inertial terms but it is, in essence, a small displacements analysis. The 3D thickness distribution is found by minimizing an error function between the static and/or modal response of the 2D and 3D monofins. The GBNM minimization algorithm carries out the identifications.

\section{Two-dimensional Modelling of the System}

The Reynolds number for the swimmer-fin system is of the order of $10^{6}$, so the viscosity is neglected. By further neglecting obstacles thickness, flow separation and water compressibility, the unsteady vortex based flow model of Le Maitre et al. ${ }^{7}$ can be used: the velocity field is obtained by the superposition of a uniform fluid flow and a field induced by vortices emitted at the fins trailing edge. The vortex intensities are calculated at each instant so that the following hold: (i) the flow remains attached along the obstacle; (ii) 
the flows from the upper and the lower surfaces join smoothly at the trailing edge (Kutta condition); (iii) the total circulation is constant. The advantage is that only the solid boundary and the wake are discretized, as opposed to meshing the whole domain, so that computer time is saved.

The swimmer is represented by 4 segments: the arms, the torso, the thighs and the tibias. The monofin is modelled by 6 rigid bars articulated by torsional springs with large rotations allowed (Fig. 2). All bars have equal and constant linear mass density. From the analysis of a monofin swimmer video the swimmer movement is approximately segment-wise harmonic:

$$
\begin{gathered}
y_{1}(t)=Y_{1}^{c}+Y_{1} \sin (2 \pi f t), \\
\theta_{i}(t)=\Theta_{i}^{c}+\Theta_{i} \sin \left(2 \pi f t-\phi_{i}\right),
\end{gathered}
$$

where $y_{1}$ is the vertical displacement of the hand, $\theta_{1}$ is the slope between the horizontal and the arms, $\phi_{1}$ is the phase angle between the vertical hand movement and the arm rotation, $\theta_{i}$ and $\phi_{i}, i=2,5$ are the angles and the phases between the segments $(i-1)$ and $i$, respectively. The parameters of Eqs. (1) and (2) (the amplitudes $Y_{1}$ and $\Theta_{i}$, the mean values $Y_{1}^{c}$ and $\Theta_{i}^{c}$, the phase angles $\phi_{i}$ and the frequency $f$ ) and the mean swimmer speed (considered to be the free-stream speed) $U_{\infty}$ are identified from measured vertical displacements of the hand, neck, shoulder, elbow, hip, knee, ankle and toe of a sprint swimmer. The following values are obtained: ${ }^{9} U_{\infty}=3.0 \mathrm{~m} / \mathrm{s}, Y_{1}=0.07 \mathrm{~m}, Y_{1}^{c}=0.0 \mathrm{~m}, \Theta_{1}=3.4^{\circ}, \Theta_{1}^{c}=-1.6^{\circ}$, $\phi_{1}=-222.6^{\circ}, \Theta_{2}=12.0^{\circ}, \Theta_{2}^{c}=1.6^{\circ}, \phi_{2}=-152.8^{\circ}, \Theta_{3}=20.0^{\circ}, \Theta_{3}^{c}=-10^{\circ}, \phi_{3}=17.2^{\circ}$, $\Theta_{4}=14.0^{\circ}, \Theta_{4}^{c}=14.0^{\circ}, \phi_{4}=17.2^{\circ}, \Theta_{5}=16.0^{\circ}, \Theta_{5}^{c}=-20.0^{\circ}$ and $\phi_{5}=107.2^{\circ}$.
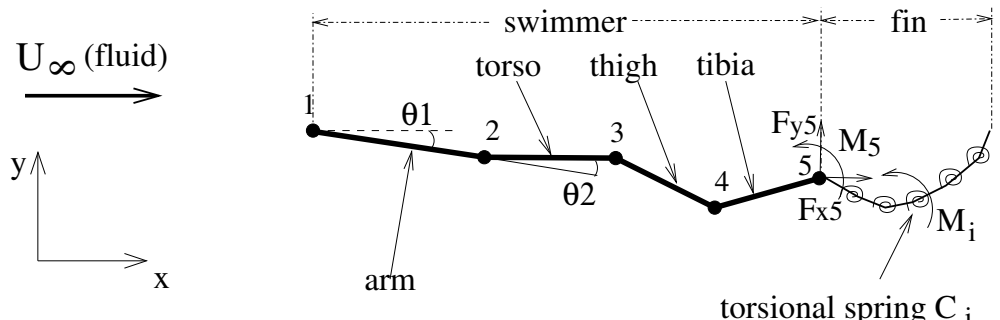

Figure 2. Swimmer and monofin representation.

The displacements of the first monofin bar $\left(\theta_{5}(t), x_{5}(t)\right.$ and $\left.y_{5}(t)\right)$ are imposed because they follow the feet. The forces distributed over the fin are obtained by means of a coupled fluid-structure calculation. The unknowns of the problem are the orientations, the angular velocities and the angular accelerations of the monofin's bar joints $\left(\theta_{i}(t), \dot{\theta}_{i}(t), \ddot{\theta}_{i}(t), i=6,10\right)$, and the efforts at the point $5\left(F_{x 5}(t), F_{y 5}(t), M_{5}(t)\right)$. The monofin dynamic equilibrium equations are solved by the Newmark time integration scheme. ${ }^{10}$ At each iteration, the system of non-linear equations is solved using a mixed Newton-Raphson/GBNM scheme. The GBNM algorithm (see Luersen et al. ${ }^{8}$ and paragraph IV,B) is employed to minimize the residue of the equations when the Newton-Raphson iterations are fruitless. A visualization of the flow, swimmer and monofin is given in Fig. 3 .

\section{Optimization Problem Formulation}

As the majority of the thrust is produced by the monofin, the purpose of the optimization is to maximize the propulsive power provided by the monofin with a constraint on the maximum total power expended by the swimmer at the fin. The time-averaged propulsive power is defined as,

$$
\bar{P}_{f x}=\frac{1}{T_{f}-T_{s}} \int_{T_{s}}^{T_{f}} \int_{0}^{L_{s}} f_{x}(s)\left|U_{\infty}\right| d s d t,
$$




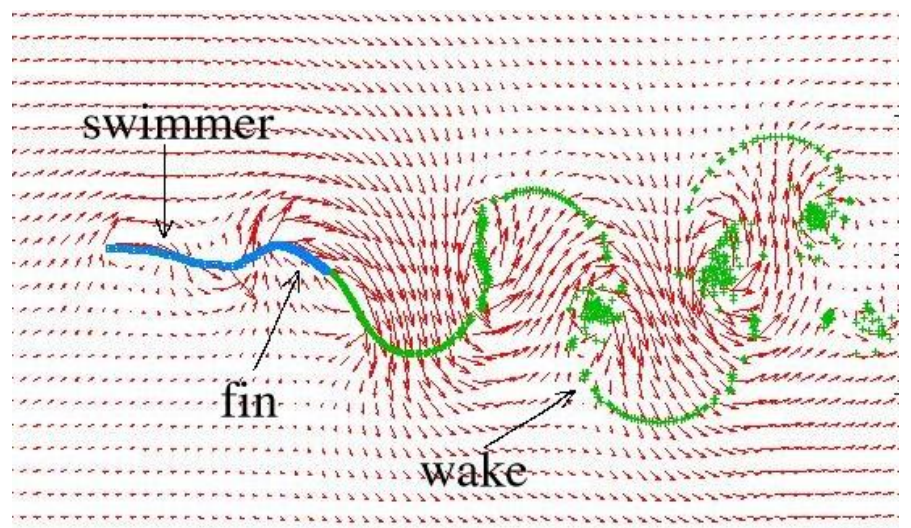

Figure 3. Flow, swimmer and monofin visualization. Continuous trace, the obstacle; $\times$, the wake particles; $\rightarrow$, the relative velocity of the fluid.

and the time-averaged power supplied by the fluid to the fin is,

$$
\bar{P}_{f}=\frac{1}{T_{f}-T_{s}} \int_{T_{s}}^{T_{f}} \int_{0}^{L_{s}}\left(f_{x}(s)\left(\dot{x}(s)+\left|U_{\infty}\right|\right)+f_{y}(s) \dot{y}(s)\right) d s d t
$$

where $U_{\infty}$ is the swimmer's mean forward speed, $\dot{x}$ and $\dot{y}$ are the velocity components of the fin in a stationary reference system with respect to the fluid speed $U_{\infty}, f_{x}(s)$ and $f_{y}(s)$ are the fluid forces per unit length over the fin at the fin natural coordinate $s, L_{s}$ is the monofin's length, $T_{s}$ and $T_{f}$ are the starting and final calculation times. Energy transmissions on the swimmers body are neglected in comparison with the fin, consequently $\bar{P}_{f}$ is considered to be the power provided to the swimmer. From the reference system that has been chosen (see Fig. 2), for a swimmer going up-stream, $\bar{P}_{f x}$ and $\bar{P}_{f}$, calculated by Eqs. (3) and (4), are negative. Thus, the objective function to be minimized is $\bar{P}_{f x}$ with a lower bound on the total power $\bar{P}_{f}$. The design variables are the torsional stiffnesses $C_{i}$. The optimization problem is formulated as,

$$
\left\{\begin{array}{l}
\min _{C_{i}} \bar{P}_{f x} \\
\text { such that, } \\
\bar{P}^{\text {min }} \leq \bar{P}_{f}, \\
C_{i}^{\text {min }} \leq C_{i} \leq C_{i}^{\text {max }}, \quad i=1,5 .
\end{array}\right.
$$

\section{Stiffness Optimization Results and Discussion}

\section{A. Parametric Studies}

In order to have an intuitive comprehension of the system behavior, parametric studies are now described where the monofin's length and spring stiffnesses change. In the first study, the monofin's length $L_{s}$ varies, while rigidities are fixed at $C_{i}=1000 \mathrm{Nm} / \mathrm{rad}$. Table 1 presents the effects on $\bar{P}_{f x}, \bar{P}_{f}$, and the power efficiency $\nu_{P}=\bar{P}_{f x} / \bar{P}_{f}$. Increasing $L_{s}$ improves $\bar{P}_{f x}$ and $\nu_{P}$, at the expense of a higher $\bar{P}_{f}$. From now on, the monofin's length is set to $0.72 \mathrm{~m}$, which is the length of the monofin used by the swimmer whose kinematics was identified.

Tables 2 and 3 summarize the effect of the rigidities $C_{i}$ on $\bar{P}_{f x}, \bar{P}_{f}$ and $\nu_{P}$. In Table 2 , all the stiffnesses are changed together. In Table 3, a reference case where $C_{i}=1000 \mathrm{Nm} / \mathrm{rad}, i=1,5$, is perturbed by setting one of the $C_{i}$ to $2000 \mathrm{Nm} / \mathrm{rad}$. 
Table 1. Effects of varying the monofin's length $L_{s}$ on the power balance.

\begin{tabular}{c|ccc}
\hline$L_{s}(m)$ & 0.5 & 0.72 & 1.0 \\
\hline $\bar{P}_{f x}(W)$ & -527.62 & -809.67 & -1147.28 \\
$\bar{P}_{f}(W)$ & -1153.30 & -1369.88 & -1747.60 \\
$\nu_{P}$ & 0.457 & 0.591 & 0.656 \\
\hline
\end{tabular}

Table 2. Effects of changing all stiffness values on the power balance.

\begin{tabular}{c|cccccc}
\hline$C_{i}, i=1,5(\mathrm{Nm} / \mathrm{rad})$ & 300 & 500 & 1000 & 5000 & 10000 & 15000 \\
\hline $\bar{P}_{f x}(W)$ & -780.38 & -749.39 & -809.67 & -1052.15 & -952.02 & -846.08 \\
$\bar{P}_{f}(W)$ & -950.34 & -1008.32 & -1369.88 & -3917.57 & -4955.79 & -5270.33 \\
$\nu_{P}$ & 0.821 & 0.743 & 0.591 & 0.269 & 0.192 & 0.161 \\
\hline
\end{tabular}

From these studies, the following pieces of information are obtained:

- Higher fin stiffness implies more power expended by the swimmer. The effect on propulsive power is not monotonous: $\bar{P}_{f x}$ first increases and then decreases with $C_{i}$;

- Moving the higher stiffness near the leading edge increases both $\bar{P}_{f x}$ and $\bar{P}_{f}$ while it decreases $\nu_{P}$;

- The efficiency $\nu_{P}$ is higher for lower fin stiffnesses;

- The propulsive power $\bar{P}_{f x}$ and the total power $\bar{P}_{f}$ are more sensitive to spring stiffnesses near the fin's leading edge than near the trailing edge.

\section{B. Optimization}

The optimization problem (Eq. (5)) is solved by means of the Globalized and Bounded Nelder-Mead algorithm. ${ }^{8}$ The GBNM is a local-global optimization method based on probabilistic restart. Local searches are performed by an improved Nelder-Mead algorithm ${ }^{11}$ where design variables can be bounded, inequality constraints taken into account by adaptive penalization, and some search failure cases prevented. The GBNM does not need gradient calculation.

The optimization problem is solved for three limits on the swimmers total power, $\bar{P}^{\text {min }}=-1400,-2000$ and $-3000 \mathrm{~W}$. As a comparison, the power measured on average distance swimmers in di Prampero et al. ${ }^{12}$ was about $1400 \mathrm{~W}$. The higher limits in the second and third cases account for the shorter distance and the 2D model that overestimates efforts (the fluid cannot go around the obstacle by the sides and there is no flow separation). The fin rigidities are bounded by: $C_{i}^{\text {min }}=300 \mathrm{Nm} / \mathrm{rad}$ and $C_{i}^{\max }=15000 \mathrm{Nm} / \mathrm{rad}$, $i=1,5$. Figure 4 shows the stiffness distributions for each optimization problem and Table 4 presents the associated power balances. The optimal stiffness distributions are tapered from the leading to the trailing edge. Changes in swimmer total power affect mainly the fin near the leading edge while low stiffness are always optimal near the trailing edge. Figure 5 shows the vertical positions of the fin's leading and trailing edges as a function of time for the optimal monofin when $\bar{P}^{\text {min }}=-2000 \mathrm{~W}$. 
Table 3. Effects of changing one spring stiffness.

\begin{tabular}{c|cccccc}
\hline$C_{1}$ & 1000 & $\mathbf{2 0 0 0}$ & 1000 & 1000 & 1000 & 1000 \\
$C_{2}$ & 1000 & 1000 & $\mathbf{2 0 0 0}$ & 1000 & 1000 & 1000 \\
$C_{3}$ & 1000 & 1000 & 1000 & $\mathbf{2 0 0 0}$ & 1000 & 1000 \\
$C_{4}$ & 1000 & 1000 & 1000 & 1000 & $\mathbf{2 0 0 0}$ & 1000 \\
$C_{5}$ & 1000 & 1000 & 1000 & 1000 & 1000 & $\mathbf{2 0 0 0}$ \\
\hline $\bar{P}_{f x}(W)$ & -809.67 & -927.97 & -832.36 & -789.65 & -790.545 & -804.12 \\
$\bar{P}_{f}(W)$ & -1369.88 & -1748.94 & -1512.46 & -1382.90 & -1354.35 & -1362.15 \\
$\nu_{P}$ & 0.591 & 0.531 & 0.550 & 0.571 & 0.584 & 0.590 \\
\hline
\end{tabular}

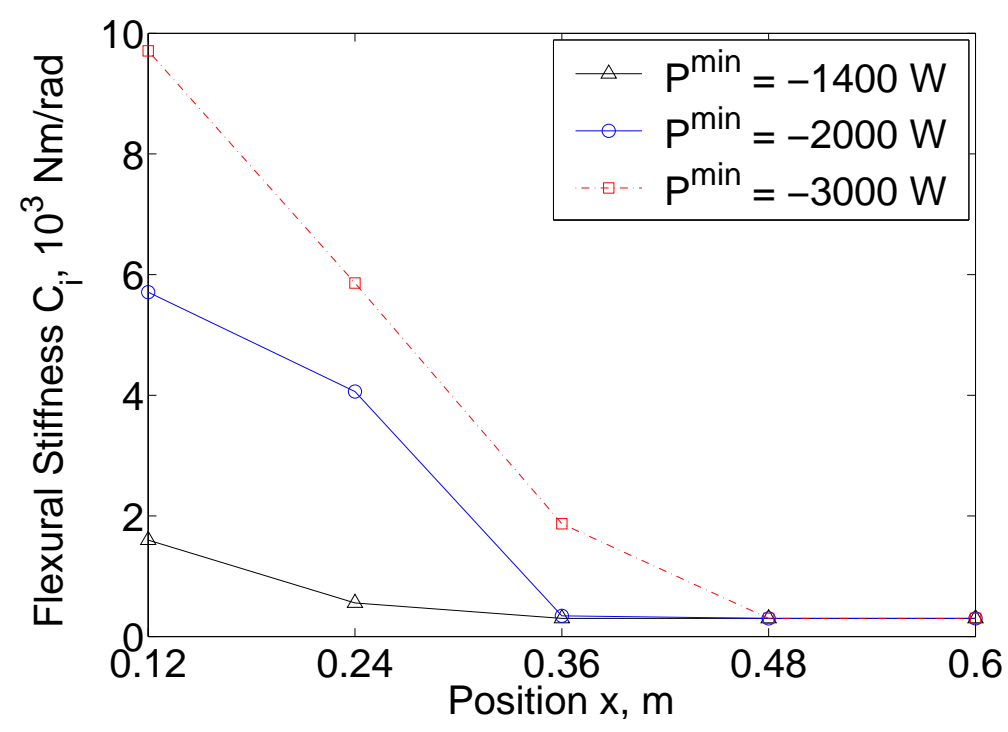

Figure 4. Optimal stiffness distributions.

\section{Translation into a 3D Structure}

The stiffness distribution obtained from the previous two-dimensional optimization is now translated into a three-dimensional structure. The mapping can be seen as an identification procedure where the "experience" is a 2D bars system whose behavior is approximated by a $3 \mathrm{D}$ finite element model of the fin. In its most general statement, this identification problem is ill-posed since the 3D system has more degrees of freedom than its 2D counterpart. Many combinations of shape and thickness distribution can represent the $2 \mathrm{D}$ monofin. In practice, however, the planform shape of the monofin is dictated by manufacturing (cost of molds) and marketing considerations which has yielded forms that mimick marine mammals. Once the fin planform shape is given, the spring stiffnesses can be mapped into a fin thickness distribution. Because the fin is manufactured using composite prepreg layup, the thickness is kept constant spanwise and varies chordwise at ply drops. The equivalence between the two models can be sought in terms of static behavior, modal behavior, or a mix of static and modal behaviors. The advantages of the static equivalence is that

$$
6 \text { of } 12
$$


Table 4. Power balances for optimal stiffness distributions.

\begin{tabular}{c|ccc}
\hline $\bar{P}^{\min }(W)$ & -1400 & -2000 & -3000 \\
\hline $\bar{P}_{f x}(W)$ & -973.15 & -1129.01 & -1208.37 \\
$\bar{P}_{f}(W)$ & -1399.99 & -1999.54 & -2988.16 \\
$\nu_{P}$ & 0.695 & 0.565 & 0.404 \\
\hline
\end{tabular}

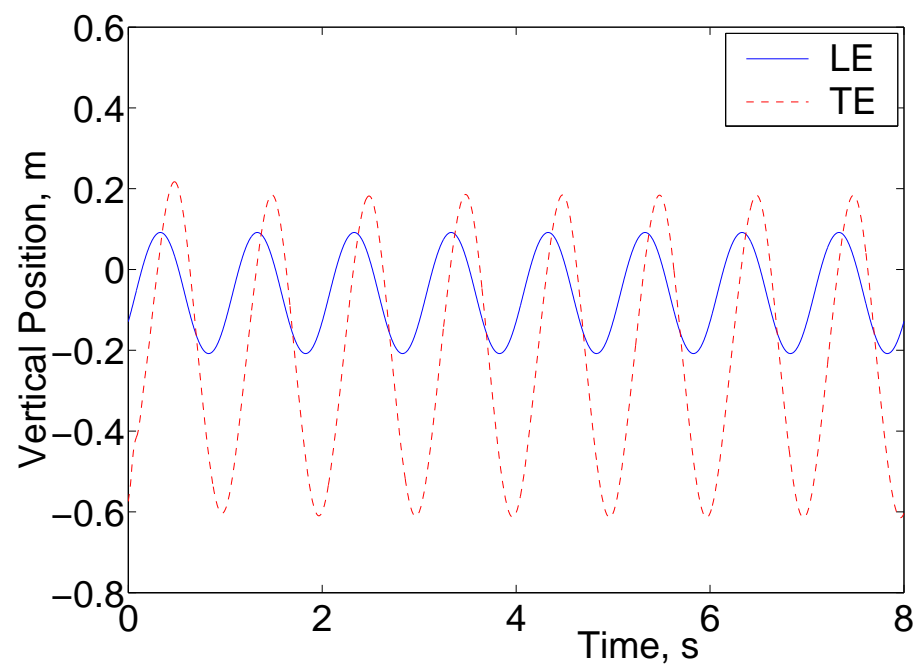

Figure 5. Evolution of the vertical positions of the fin's leading and trailing edges (LE and TE) for the optimal monofin under the constraint $\bar{P}^{\text {min }}=-2000 \mathrm{~W}$.

large displacements analyses may be available. However, it neglects the fin inertia in comparison to water inertia and fin flexural stiffness. On the contrary, the modal dynamic identification accounts for both fin inertia and flexural stiffness but it is, in essence, a small displacements analysis. Furthermore, 3D nonbending modes have no pendant in the 2D system, and the higher natural bending frequencies are far away from the frequency of the imposed movement. Hence, the first natural bending mode is more relevant than other natural bending modes. For these reasons, only the first natural mode, which has empirically been found on the monofin to consistently be bending, is considered. The 3D thickness distribution is found by minimizing

$$
J=\alpha J_{\text {static }}+(1-\alpha) J_{\text {freq }},
$$

where,

$$
J_{\text {static }}=\frac{\sum_{i=1}^{N C P}\left(\left(u_{i}-\tilde{u}_{i}\right)^{2}+\left(v_{i}-\tilde{v}_{i}\right)^{2}\right)}{\sum_{i=1}^{N C P}\left(u_{i}^{2}+v_{i}^{2}\right)} \text { and } J_{\text {freq }}=\frac{\left|w_{1}^{2}-\tilde{w}_{1}^{2}\right|}{w_{1}^{2}} .
$$

$\alpha$ is a weight factor that balances contributions from static and modal criteria, $\left(u_{i}, v_{i}\right)$ are the target displacements at the bar joints of the simplified model, $\left(\tilde{u}_{i}, \tilde{v}_{i}\right)$ are the displacements at the $N C P$ corresponding 
control points of the 3D finite element model, $w_{1}$ is the target first natural circular frequency of the simplified model and $\tilde{w}_{1}$ is the first natural circular frequency of the finite element model.

The 3D fin is analyzed with a finite element model which relies on solid elements improved for thin structures. ${ }^{13}$ The parameterization of the fin is described in Fig. 6.

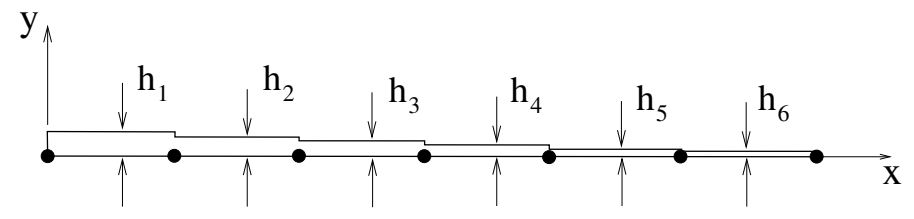

(a)

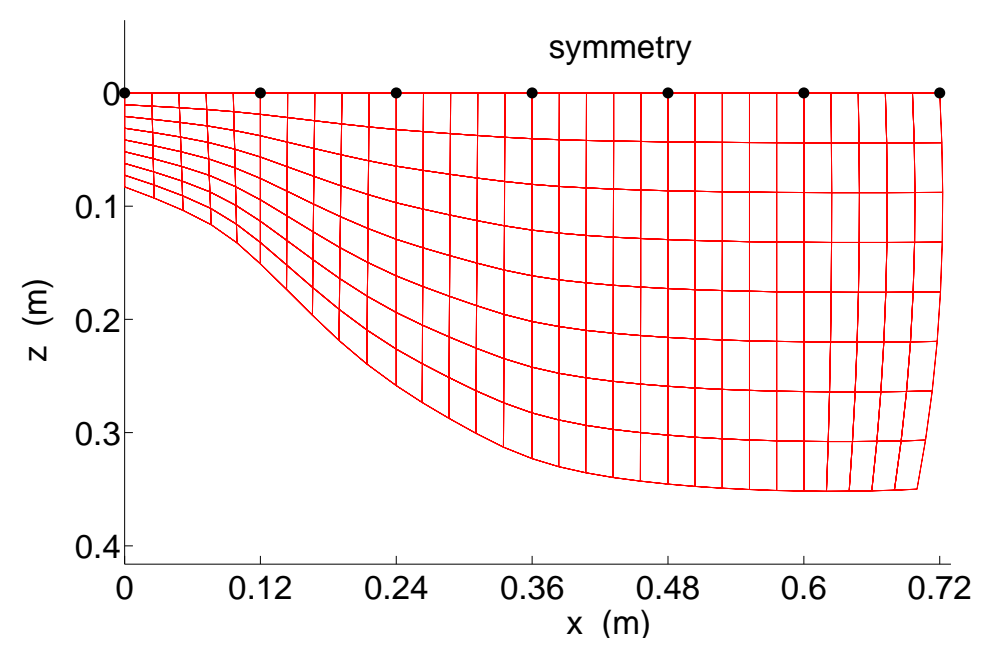

(b)

Figure 6. Fin parameterization: thickness profile (a), half-planform shape and finite element mesh (b). The dots represent the $N C P$ points where $J_{\text {static }}$ is evaluated.

The final thickness distribution is identified by solving,

$$
\left\{\begin{array}{l}
\min _{h_{i}} J \\
\text { such that, } \\
h_{i}^{\text {min }} \leq h_{i} \leq h_{i}^{\text {max }}, \quad i=1,6 \text { and } \\
h_{i} \leq h_{i-1}, \quad i=2,6
\end{array}\right.
$$

where the thicknesses $h_{i}$ are bounded by $h_{i}^{\text {min }}=1.5 \times 10^{-4} \mathrm{~m}$ and $h_{i}^{\max }=2 \times 10^{-2} \mathrm{~m}, i=1,6$. The constraints $h_{i} \leq h_{i-1}, i=2,6$, are handled by reordering the variables $h_{i}$ in the finite element (FE) analysis but keeping them unordered in the optimization. It was observed on the current problem that this reordering is more efficient than the adaptive penalization of the GBNM optimizer. 


\section{Identification Results and Discussion}

Firstly, when $J=J_{\text {static }}(\alpha=1)$, the difference between the small and large displacements analyses, for a given load case, is shown. A uniformly distributed load $p$ is applied on the fin surface and the "foot" $(x=0)$ is clamped. It should be noted that different load cases do not generate different solutions (at the condition that the equilibrium relative angles are non-null). ${ }^{14}$

Secondly, the problem is solved for a mixed static and modal criterion $(\alpha=0.5)$.

It was found that the 2D model has a dynamic behavior which cannot be reproduced by $3 \mathrm{D}$ fins because $2 \mathrm{D}$ and $3 \mathrm{D}$ models do not present the same mass distribution. The 2D model has a constant chordwise mass density and 3D fins have a tapered chordwise thickness distribution and a varying width that induce a varying chordwise mass distribution. So the solution for $\alpha=0\left(J=J_{\text {freq }}\right)$ is not completely realistic. Also, it was numerically observed that the dynamic formulation presents many solutions (many thickness distributions) that are very close. ${ }^{14}$

The thickness identification is performed for the optimal flexural distribution when $\bar{P}^{\text {min }}=-2000$. The GBNM algorithm is used to solve problem (8). In order to preserve the confidentiality of the final design, the thicknesses are normalized by the maximum thickness and the fin material properties are not given.

Figure 7 compares identified thickness distribution solutions to the mixed $(\alpha=0.5)$, the static linear (small displacements and $\alpha=1$ ), and the static non-linear (large displacements and $\alpha=1$ ) formulations.

For the static small displacements formulation the load is $p=4.0875 \mathrm{~N} / \mathrm{m}$. For the static large displacements formulation the load distribution used in the small displacements identification is kept but the intensity is six times higher in order to generate a large displacements problem: $p=24.525 \mathrm{~N} / \mathrm{m}$. Because the discrepancies in identified thicknesses between large and small displacements are negligible, the static analysis of the mixed criterion is under small displacements.

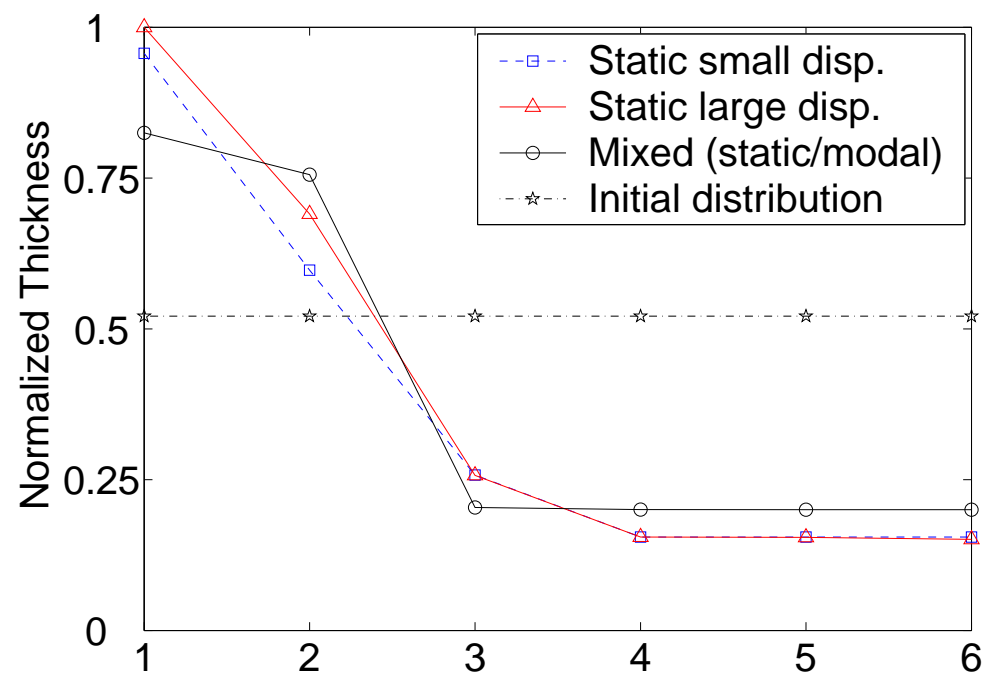

Figure 7. Comparison of the solutions (thickness distributions) of the three identification formulations.

Table 5 compares the first natural frequencies of the 2D and 3D models obtained with the three formulations. The reader should keep in mind that frequencies are not accounted for in the static identifications.

Figures 8 and 9 show the deformed shapes of the 2D bars model and the mid-plane symmetry line $(z=0)$

9 of 12

American Institute of Aeronautics and Astronautics 
Table 5. First natural frequencies comparison, in $h z$.

\begin{tabular}{cccc}
\cline { 2 - 4 } 2D bars model & \multicolumn{3}{c}{ 3D monofin } \\
& $\begin{array}{c}\text { Static Small } \\
\text { Displacements }\end{array}$ & $\begin{array}{c}\text { Static Large } \\
\text { Displacements }\end{array}$ & Mixed Criterion \\
\hline 1.5400 & 2.4842 & 2.5584 & 2.2115 \\
\hline
\end{tabular}

of the FE model built with the identified thicknesses for the static small displacements formulation and the mixed formulation, respectively.

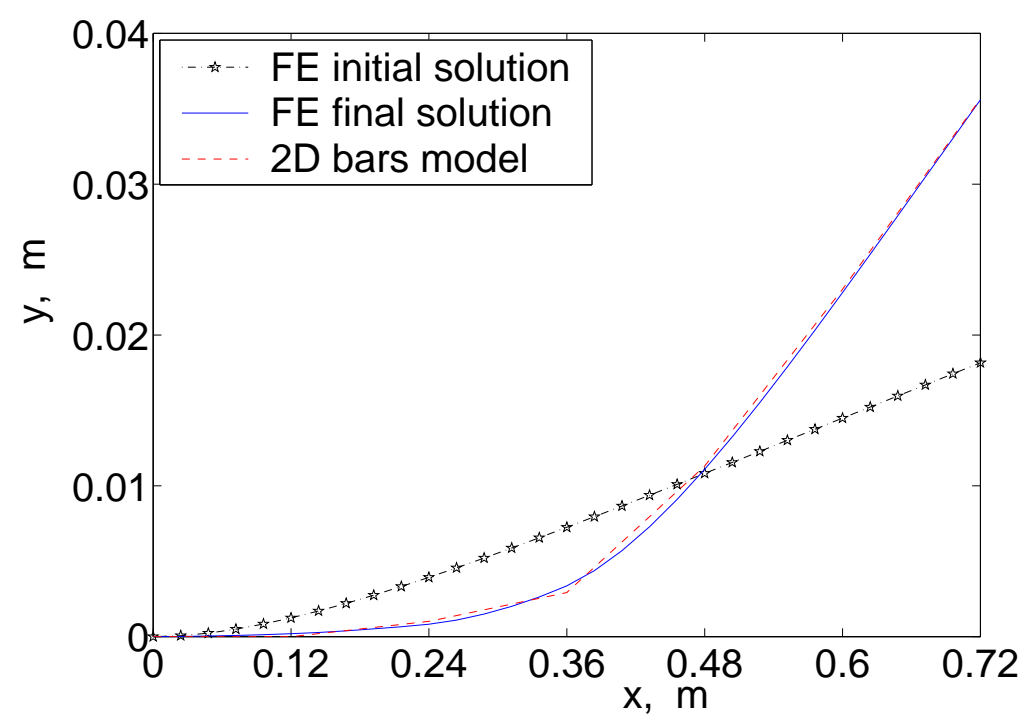

Figure 8. Deformed shapes comparison where the FE model is identified using the static small displacements formulation.

The solutions in small and large displacements are close, with large displacements solutions being thicker near the monofin's leading edge (see Fig. 7). This is explained by the fact that large displacements analysis generates larger vertical displacements in the clamped region. Comparing mixed formulation to the static analysis formulations, material is removed from the clamped region and added at the tip. This provides lower first natural frequency, which is closer to the 2D model. This is traded against a slight departure from the targetted static deformed shape.

\section{Conclusion}

A strategy for optimizing a swimming monofin has been presented. It is based on the optimization of a simplified two-dimensional swimmer-fin-fluid model followed by a mapping of the result into a threedimensional structure. The two-dimensional model consists of an unsteady vortex based fluid model in dynamic equilibrium with a bar system representing the fin. It is numerically efficient and can be included into an optimization loop. The flexural distribution over the bar system is optimized by maximizing the propulsive power with a bound on the total power. The optimal stiffness distributions are tapered from the 


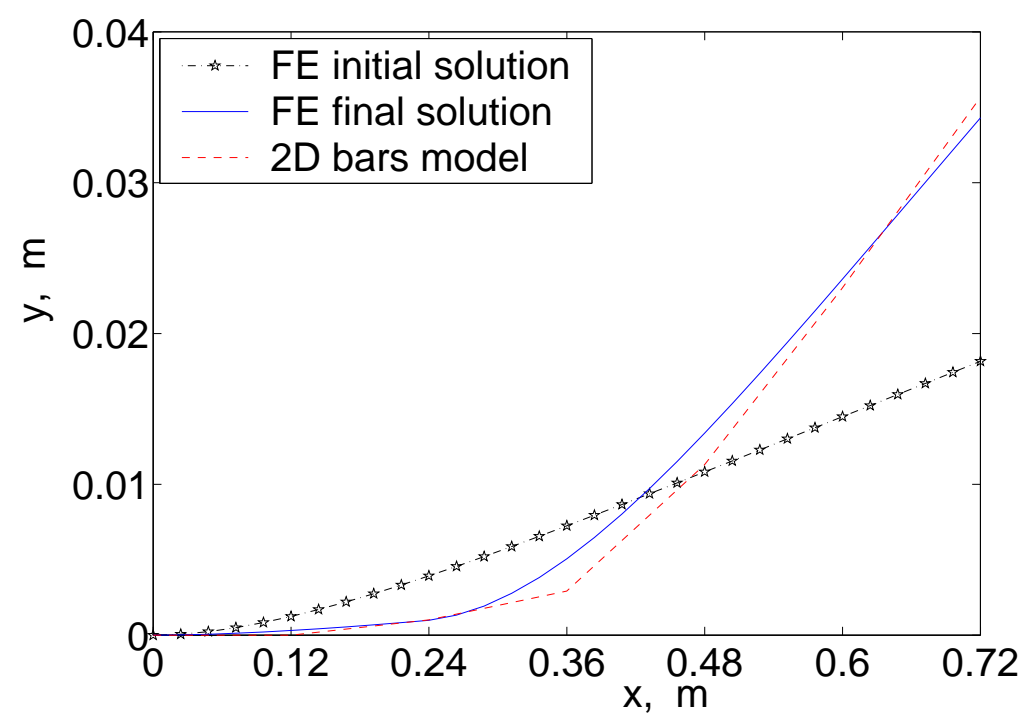

Figure 9. Deformed shapes comparison where the FE model is identified using the mixed static/dynamic formulation.

leading to the trailing edge.

The solution has then been translated into a 3D monofin structure. The shape and the material of the monofin are fixed by manufacturing constraints. The thickness distribution is identified to be statically and/or dynamically equivalent to the optimized 2D system. The influence of the various formulations on the final thickness distributions have been studied.

It is recommended to choose the mixed static/dynamic formulation when translating a 2D fin design into 3D. Indeed, the static and dynamic formulations do no yield the same designs. In terms of static analysis, a small displacements finite element model is sufficient. It is observed that small and large displacements formulations do not present significant design differences. Moreover, when mixed formulation is considered, a highly accurate static analysis is not essential because static and dynamic equivalences between the 2D and 3D models are traded-off, so that there is no longer a precise deformed shapes match.

In both the optimization and identification steps, the GBNM algorithm has performed the minimizations.

\section{Acknowledgments}

Fundings for this work were provided by $\mathrm{CNPq}$ Brazilian research agency for M. A. Luersen and by the Breier fin company, France.

\section{References}

\footnotetext{
${ }^{1}$ Sfakiotakis, M., Lane, D.M. and Davies, B.C., "Review of Fish Swimming Modes for Aquatic Locomotion", IEEE J. of Oceanic Eng., 24(2), 1999, pp. 237-252.

${ }^{2}$ Pedro, G., Suleman, A. and Djilali, N., "A numerical study of the propulsive efficiency of a flapping hydrofoil", International Journal for Numerical Methods in Fluids, 42, 2003, pp. 493-526.

${ }^{3}$ Anderson, J.M., Streitlien, K., Barret, D.S., Triantafyllou, M.S., "Oscillating foils of high propulsive efficiency", Journal of Fluids Mechanics, 360, 1998, pp. 41-72.
}

11 of 12 
${ }^{4}$ Zamparo, P., Pendergast,D.R., Termin, B., Minetti, A.E., "How fins affect the efficiency and economy of human swimming", The International Journal of Experimental Biology, 205, 2002, pp. 2665-2676.

${ }^{5}$ Baly, L., Durey, A. and Favier, D., "Etude des fréquences et amplitudes ondulatoires chez le nageur avec palmes de haut niveau", Journées de l'Ecole Doctorale des Sciences de la Vie et de la Santé, LABM, UMSR 2164, CNRS, Univ. de Méditerranée, France (http://www.ujf-grenoble.fr/ufraps/acaps/Actes/Poster/baly.pdf) (in French).

${ }^{6}$ Kvamsdal, T., Jenssen, C., Okstad, K. and Amundsen, J., "Fluid-structure interaction for structural design", Proceedings of the International Symposium on Computational Methods for Fluid-Structure Interaction (FSI'99), edited by Kvamsdal et al., Tapir Publishers, Trondheim, Norway, February 1999, pp. 211-238.

${ }^{7}$ Le Maître, O., Huberson, S. and Souza de Cursi, E., "Unsteady model of sail and flow interaction", Journal of Fluids and Structures, 13, 1999, pp. 37-59.

${ }^{8}$ Luersen, M.A., Le Riche, R. and Guyon, F., "A constrained, globalized, and bounded Nelder-Mead method for engineering optimization", Structural and Multidisciplinary Optimization, Springer, Vol. 27, 2004, pp. 43-54.

${ }^{9}$ Luersen, M.A., Le Riche, R., Le Maître, O. and Breier, E., "Optimisation de monopalmes de nage", Proceedings of the 6th Colloque National en Calcul des Structures, Vol. 3, CSMA/AFM publ., Giens, France, May 2003, pp. 207-214 (in French).

${ }^{10}$ Géradin, M., Cardona, A., Flexible Multibody Dynamics: A Finite Element Approach. John Wiley \& Sons, Inc., New York, USA, 2001.

${ }^{11}$ Nelder, J.A., and Mead, R., "A simplex for function minimization", Computer J., 7, 1965, pp. 308-313.

${ }^{12}$ di Prampero, P.E., Pendergast, D.R., Wilson, D.W., Rennie, D.W., "Energetics of swimming in man", Journal of Applied Physiology, Vol. 360, No. 1, 1974, pp. 1-5.

${ }^{13}$ Lemosse, D., Dhatt, G., "Study of Shell-like Structures by Brick Finite Element Model", Proceedings of The 5th International Conference on Computational Structures Technology, Vol. H, Leuven, Belgium, 6-8 September 2000, pp. 183-190.

${ }^{14}$ Luersen, M.A., Le Riche, R, Lemosse, D., "Identification of a 3D Shape from a 2D Design: Application to a Swimming Monofin", Accepted to be published in the Proceedings of the 4 th International Conference on Engineering Computational Technology, Lisbon, Portugal, 7-9 September 2004. 